\title{
ON THE FLUCTUATIONS OF THE SINR AT THE OUTPUT OF THE WIENER FILTER FOR NON CENTERED CHANNELS: THE NON GAUSSIAN CASE
}

\author{
Abla Kammoun ${ }^{1}$, Malika Kharouf ${ }^{1}$, Romain Couillet ${ }^{2}$, Jamal Najim ${ }^{1}$ and Mérouane Debbah ${ }^{2}$ \\ Télécom ParisTech 46, rue Barrault, 75634 Paris Cedex 13, France ${ }^{1}$ \\ Supélec ${ }^{2}$
}

\begin{abstract}
In the context of multidimensional signals, the linear Wiener receiver is frequently encountered in wireless communication and in array processing; it is in fact the linear receiver that achieves the lowest level of interference. In this contribution, we focus on the study of the associated Signal-to-interference plus noise ratio (SINR) at its output in the context of Ricean multiple-input multiple-output (MIMO) channels. The case of Ricean channels, which induces non-centered random variables, can be encountered in several practical environments and has not been studied so far, as it raises substantial technical issues. With the help of large random matrix theory, which has shown to be fruitful to successfully address several problems in wireless communications, we study the behaviour of the SINR, together with its fluctuations via a central limit theorem. As realistic models also involve non-Gaussian random variables, we relax the Gaussian assumption. This results in an extra term involving the fourth cumulant in the expression of the variance.
\end{abstract}

Index Terms - Central Limit Theorem, MIMO systems, Quadratic Form, Random Matrix Theory, Wiener Filtering

\section{INTRODUCTION}

In the mid-nineties, Telatar[1] and Foshini[2] demonstrated the great potential of the multiple-input multiple-output (MIMO) technology to meet the increasing demand to higher data rates. Indeed, their analyses show that the mutual information over an $N \times n$ MIMO channel is proportional to $\min (N, n)$. Nevertheless, the data rates stipulated by the mutual information are only achievable in practice through the use of high complexity algorithms. In reality, one usually uses suboptimal decoders such as the Wiener filter, whose performance are clearly of interest. The SINR at its output has been extensively studied in the literature, for the correlated and non correlated cases, $[3,4,5]$. The non-centered Gaussian case has been considered in [6], where only the first order result has been provided.

In this paper, we extend previous works to the case when the channel, not necessarily Gaussian, admits a deterministic line-of-sight component. Allowing the random compo- nent to be non-Gaussian improves the fit of the presented results to the true SINR in many practical scenarios, especially encountered in severe fading situations, like for instance the Nakagami-m channels particularly suited to model some urban multipath environments [7]. In particular, it is proved that in the asymptotic regime (i.e. for $N, n \rightarrow+\infty$ at the same pace), the SINR fluctuates around its first order approximate as a centered Gaussian random variable whose variance depends on the cumulant of the entries and the deterministic line-of-sight matrix.

Notations: In the following, boldface lower case symbols represent vectors, capital boldface characters denote matrices $\left(\mathbf{I}_{N}\right.$ is the size- $N$ identity matrix). The transpose and Hermitian transpose operators are denoted $(\cdot)^{\top}$ and $(\cdot)^{*}$, respectively. The expectation and inverse operators will be denoted by $\mathbb{E}$ and $(.)^{-1}$.

\section{SYSTEM MODEL AND PROBLEM SETTING}

Consider a wireless MIMO transmission model with $N$ antennas at the reception side and $n+1$ antennas at the transmission side corresponding to $n+1$ distant sources. Let $\mathbf{H}$ denote the $N \times n+1$ channel matrix given by:

$$
\mathbf{H}=\frac{1}{\sqrt{n}}\left(\sqrt{\frac{1}{K+1}} \mathbf{X}+\sqrt{\frac{K}{K+1}} \widetilde{\mathbf{A}}\right),
$$

where $K$ is called the Rice factor, $\mathbf{X}$ is a random matrix with i.i.d. entries with zero mean and unit variance (not necessarily Gaussian), and $\widetilde{\mathbf{A}}$ is the deterministic matrix which stands for the line-of-sight component assumed known to the receiver. Consider the following transmission model:

$$
\mathbf{r}=\mathbf{H} \mathbf{s}+\mathbf{n}
$$

where $\mathbf{n}$ is the additive white Gaussian noise (AWGN) verifying $\mathbb{E} \mathbf{n}=\mathbf{0}$ and $\mathbb{E} \mathbf{n n}^{*}=\rho \mathbf{I}_{N}$, and $\mathbf{s}=\left[s_{0}, \cdots, s_{n}\right]$ is the unknown random vector of transmitted symbols with size $n+1$. Partition the channel matrix

$$
\mathbf{H}=\left[\mathbf{y}+\sqrt{\frac{K}{K+1}} \mathbf{a}, \quad \mathbf{Y}+\sqrt{\frac{K}{K+1}} \mathbf{A}\right]
$$


, then the estimate of $s_{0}$ at the LMMSE receiver $\widehat{s}_{0}$ reads:

$$
\widehat{s}_{0}=\left(\mathbf{y}^{*}+\mathbf{b}^{*}\right)\left((\mathbf{Y}+\mathbf{B})\left(\mathbf{Y}^{*}+\mathbf{B}^{*}\right)+\rho \mathbf{I}_{N}\right)^{-1} \mathbf{r},
$$

where $\mathbf{b}=\sqrt{\frac{K}{K+1}}$ a and $\mathbf{B}=\sqrt{\frac{K}{K+1}} \mathbf{A}$. The SINR $\beta_{n}$ is thus given by:

$$
\beta_{n}=\left(\mathbf{y}^{*}+\mathbf{b}^{*}\right) \mathbf{Q}(\mathbf{y}+\mathbf{b}),
$$

where $\mathbf{Q}$ is the matrix given by:

$$
\mathbf{Q}=\left((\mathbf{Y}+\mathbf{B})\left(\mathbf{Y}^{*}+\mathbf{B}^{*}\right)+\rho \mathbf{I}_{N}\right)^{-1} .
$$

For fixed dimensions $N, n$, the study of $\beta_{n}$ is rather difficult. In what follows, we will consider the asymptotic regime defined as $N, n \rightarrow \infty$ such that:

$$
0<\liminf \frac{N}{n} \leq \limsup \frac{N}{n}<+\infty,
$$

which we will denote $n \rightarrow+\infty$ for notational simplicity. In this regime, the first and second order statistics of $\beta_{n}$ will depend on the following deterministic quantities which we recall hereafter:

Theorem 1 ([8]). For any $\rho>0$, the deterministic system:

$$
\left\{\begin{array}{l}
\delta(\rho)=\frac{1}{n} \operatorname{Tr} \mathbf{T}_{n}(\rho) \\
\widetilde{\delta}(\rho)=\frac{1}{n} \operatorname{Tr} \widetilde{\mathbf{T}}_{n}(\rho)
\end{array}\right.
$$

where $\mathbf{T}_{n}$ and $\widetilde{\mathbf{T}}_{n}$ are the matrices

$$
\left\{\begin{array}{l}
\mathbf{T}_{n}(\rho)=\left(\rho(K+1)(1+\widetilde{\delta}(\rho)) \mathbf{I}_{N}+\frac{K \mathbf{A A}^{*}}{(1+\delta(\rho))}\right)^{-1} \\
\widetilde{\mathbf{T}}_{n}(\rho)=\left(\rho(K+1)(1+\delta(\rho)) \mathbf{I}_{n}+\frac{K \mathbf{A}^{*} \mathbf{A}}{(1+\widetilde{\delta}(\rho))}\right)^{-1}
\end{array}\right.
$$

admits a unique solution $(\delta, \widetilde{\delta})$ in $(0, \infty)^{2}$.

\section{FIRST ORDER RESULT}

Theorem 2. Assume that $\sup _{n}\|\mathbf{A}\|<\infty$, where $\|$.$\| is the$ spectral norm. In the asymptotic regime, the following holds true:

$$
\beta_{n}-\bar{\beta}_{n} \underset{n \rightarrow+\infty}{\longrightarrow} 0 \text { almost surely }
$$

where

$$
\bar{\beta}_{n}=\frac{1}{n} \operatorname{tr} \mathbf{T}_{n}+K \mathbf{a}^{*} \mathbf{T}_{n} \mathbf{a},
$$

where $\mathbf{T}_{n}$ is given in theorem 1 .

Proof. To prove Theorem 2, we shall decompose $\beta_{n}$ as follows

$$
\beta_{n}=\mathbf{b}^{*} \mathbf{Q b}+\mathbf{b}^{*} \mathbf{Q} \mathbf{y}+\mathbf{y}^{*} \mathbf{Q b}+\mathbf{y}^{*} \mathbf{Q y} .
$$

It suffices then to determine the asymptotic limit of each of the fourth terms. From theorem 1.1 in [9], we know that:

$$
\mathbf{b}^{*} \mathbf{Q b}-(K+1) \mathbf{b}^{*} \mathbf{T b} \underset{n \rightarrow \infty}{\longrightarrow} 0 \text { almost surely. }
$$

Hence, substituting b by $\sqrt{\frac{K}{K+1}}$ a, we get:

$$
\mathbf{b}^{*} \mathbf{Q b}-K \mathbf{a}^{*} \mathbf{T a} \underset{n \rightarrow \infty}{\longrightarrow} 0 \text { almost surely. }
$$

Besides, by the strong law of large numbers for weighted independent random variables, the crossed terms converge to zero almost surely [10]. Finally, using standard results of the characterization of the convergence of quadratic forms (see Lemma 2.7 in [11]), one can easily establish that:

$$
\mathbf{y}^{*} \mathbf{Q y}-\frac{1}{n(K+1)} \operatorname{tr} \mathbb{E} \mathbf{Q} \underset{n \rightarrow \infty}{\longrightarrow} 0 \text { almost surely. }
$$

Finally, the theorem follows from the fact that:

$$
\frac{1}{n(K+1)} \operatorname{tr}(\mathbb{E} \mathbf{Q})-\frac{1}{n} \operatorname{tr} \mathbf{T} \underset{n \rightarrow \infty}{\longrightarrow} 0 .
$$

\section{SECOND ORDER RESULT}

Beyond the convergence $\beta_{n}-\bar{\beta}_{n} \rightarrow 0$, a natural question arises regarding the accuracy of $\bar{\beta}_{n}$ for finite values of $N, n$. This can provide insights about the outage probability which is defined as the probability that the SINR falls below a certain threshold. To answer to this question, one needs to study the fluctuations which will be described in the following theorem:

Theorem 3. Let $\gamma=\frac{1}{n} \operatorname{Tr}\left(\mathbf{T}^{2}\right), \quad \widetilde{\gamma}=\frac{1}{n} \operatorname{Tr}\left(\widetilde{\mathbf{T}}^{2}\right), \mathbf{S}=$ $\operatorname{diag}(\mathbf{T})$, and $\widetilde{\mathbf{S}}=\operatorname{diag}(\widetilde{\mathbf{T}})$. Let $\kappa$ be the fourth cumulant of the entries of $\mathbf{X}$ given by $\kappa=\mathbb{E}\left|X_{1,1}\right|^{4}-2$. Define $\Delta_{n}, \alpha_{n}$ and $\xi_{n}$ as:

$$
\begin{aligned}
\Delta_{n} & =\left(1-\frac{K}{n(1+\delta)^{2}} \operatorname{Tr}\left(\mathbf{A} \mathbf{A}^{*} \mathbf{T}^{2}\right)\right)^{2}-\rho^{2}(K+1)^{2} \gamma \tilde{\gamma} \\
\alpha_{n} & =\frac{1}{\gamma(1+\delta)^{4}}\left\{\gamma\left(\frac{K}{n} \operatorname{tr} \mathbf{T}^{2} \mathbf{A} \mathbf{A}^{*}+K^{2} \mathbf{a}^{*} \mathbf{T} \mathbf{A} \mathbf{A}^{*} \mathbf{T a}\right)\right. \\
& \left.+\left((1+\delta)^{2}-\frac{K}{n} \operatorname{tr} \mathbf{T}^{2} \mathbf{A} \mathbf{A}^{*}\right)\left(\gamma+K \mathbf{a}^{*} \mathbf{T}^{2} \mathbf{a}\right)\right\}^{2}, \\
\xi_{n} & =\rho^{2}(K+1)^{2} K^{2} \frac{1}{n} \operatorname{tr} \tilde{\mathbf{S}}^{2} \sum_{k=1}^{N}\left[\mathbf{T} \mathbf{a} \mathbf{a}^{*} \mathbf{T}\right]_{k, k}^{2} \\
& +\frac{K^{4}}{(1+\delta)^{4}} \sum_{k=1}^{N}\left|\mathbf{u}^{*} \mathbf{T} \mathbf{a}_{k} \mathbf{a}_{k}^{*} \mathbf{T u}\right|^{2} \frac{1}{n} \operatorname{tr} \mathbf{S}^{2}+\frac{1}{n} \operatorname{tr} \mathbf{S}^{2} .
\end{aligned}
$$

Then, the following holds true:

1. The sequence of real numbers:

$$
\Omega_{n}^{2}=\frac{\alpha_{n}}{\Delta_{n}}-\frac{K^{2}\left(\mathbf{a}^{*} \mathbf{T}^{\mathbf{2}} \mathbf{a}\right)^{2}}{\gamma}+\kappa \xi_{n}
$$


satisfies:

$$
0<\lim \inf _{n} \Omega_{n}^{2} \leq \lim \sup _{n} \Omega_{n}^{2}<+\infty
$$

\section{The SINR $\beta_{n}$ satisfies:}

$$
\sqrt{\frac{n}{\Omega_{n}^{2}}}\left(\beta_{n}-\mathbb{E} \beta_{n}\right) \underset{n \rightarrow \infty}{\longrightarrow} \mathcal{N}(0,1), \text { in distribution. }
$$

The technical proofs of our results rely on the REFORM (REsolvent, FORmula and Martingale) method which has been successfully used to establish the CLT for the mutual information and the SINR for centered channels. It merely consists in decomposing $\beta_{n}-\mathbb{E} \beta_{n}$ into a some of difference of martingales by choosing the appropriate filtration. Details are omitted because of lack of space, but the interested reader can refer to the works in [5] and [12].

Remark 1. The expressions of the asymptotic theoretical variance might seem involved. Their numerical computation is quite easy since it merely depends on the system solutions $\delta$ and $\tilde{\delta}$ which can be computed using standard iterative algorithms.

Remark 2. Theorem 3 describes the asymptotic behavior of the SINR around its expected moment. Determining the fluctuations of the SINR around the deterministic approximate $\bar{\beta}_{n}$ is not immediate since according to the results in [9], $\sqrt{n}\left(\mathbb{E} \beta_{n}-\bar{\beta}_{n}\right)=\mathcal{O}(1)$. We prove hereafter a stronger result which states that $\sqrt{n} \mathbb{E}\left(\beta_{n}-\bar{\beta}_{n}\right)$ tends in reality to zero.

Theorem 4. Under mild technical assumptions, the SINR satsfies:

$$
\mathbb{E} \beta_{n}-\bar{\beta}_{n} \underset{n \rightarrow \infty}{\longrightarrow} 0
$$

As a corollary, we get thus:

Corollary 1. In the asymptotic regime, and under mild technical assumptions, the SINR satisfies:

$$
\sqrt{\frac{n}{\Omega_{n}^{2}}}\left(\beta_{n}-\bar{\beta}_{n}\right) \underset{n \rightarrow \infty}{\longrightarrow} \mathcal{N}(0,1), \text { in distribution. }
$$

\section{SIMULATIONS}

In this section, we check by simulations the accuracy of our results. We assume a non-centered channel with a line of sight matrix $\widetilde{\mathbf{A}}=\left[\mathbf{a}\left(\alpha_{1}\right), \cdots, \mathbf{a}\left(\alpha_{n+1}\right)\right]$ where $\mathbf{a}(\alpha)=$ $\left[1, e^{\jmath \alpha}, \cdots, e^{\jmath(N-1) \alpha}\right]^{\top}$ is a directional vector, the $\alpha_{i}$ being some given phase variables. The entries of the non-line of sight matrix $\mathbf{X}$ are assumed to satisfy $X_{i, j}=r_{i, j} \exp \left(j \theta_{i, j}\right)$, where $\theta_{i, j}$ are i.i.d. uniform phase variables over $[0,2 \pi]$ and $r_{i, j}$ are i.i.d. positive random variables. Depending on the distribution of $r_{i, j}$, many types of channels can be modelled.
We consider here the Nakagami-m channel, for which the distribution of $r_{i, j}$ is given by:

$$
f_{\mu, \omega}(x)=\frac{2 \mu^{\mu}}{\Gamma(\mu) \omega^{\mu}} x^{2 \mu-1} e^{-\frac{\mu}{\omega} x^{2}},
$$

where $\omega$ is set to 1 in order to get $\mathbb{E}\left|X_{1,1}\right|^{2}=1$. The cumulant of $X_{i, j}$ is thus given by: $\kappa=1+\frac{1}{\mu}-2$. It should be also noted that the Rayleigh distribution corresponding to the Rayleigh channel (Gaussian non-line of sight component) can be retrieved by setting $\mu$ to 1 .

Fig. 1 displays the empirical estimation of $\mathbb{E} \beta_{n}$ as well as $\bar{\beta}_{n}$ with respect to $c=\frac{N}{n}$ when $n=32, N$ ranging from 4 to 32 and the rice factor and $\rho$ set to $K=1, \rho=0.5$. In that experiment, we consider the case of Gaussian random variables $(\mu=1)$, since the asymptotic approximate of $\mathbb{E} \beta_{n}$ does not depend on the cumulant. As expected, the SINR increases when the number of receiving antennas grows thereby increasing the channel diversity.

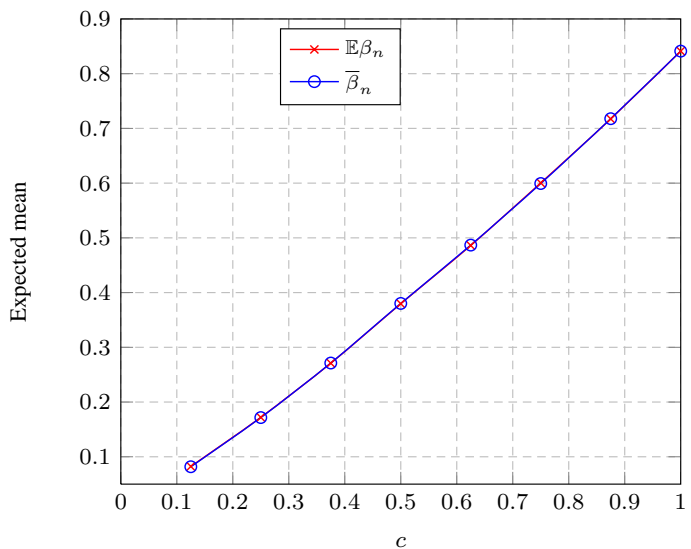

Fig. 1. Empirical and asymptotic means with respect to the $c$.

In a second experiment, we study the effect of the rice factor on the variance of the SINR. Fig. 2 displays the variance of the SINR for $n=32, N=8, \rho=0.5$ and $\mu=0.6$, while the rice factor $K$ ranges from 0.1 to 5 . Note that when $K$ grows, the variance decreases, thus reducing the fluctuations of the SINR. Finally, we represent in fig. 3 the histogram of $\sqrt{\frac{n}{\Omega_{n}^{2}}}\left(\beta_{n}-\bar{\beta}_{n}\right)$ when $K=2, \rho=1, N=16$ and $n=32$. We notice that the behaviour of the SINR around its asymptotic equivalent is similar to that of a Gaussian random variable.

\section{CONCLUSION}

In this paper, we have established the asymptotic Gaussian behavior of the SINR at the output of the LMMSE receiver for non-centered MIMO channels. We have provided simulations that support our theoretical claims.

\section{REFERENCES}

[1] I.E. Telatar, "Capacity of Multi-Antenna Gaussian Channels," published in European Transactions on 


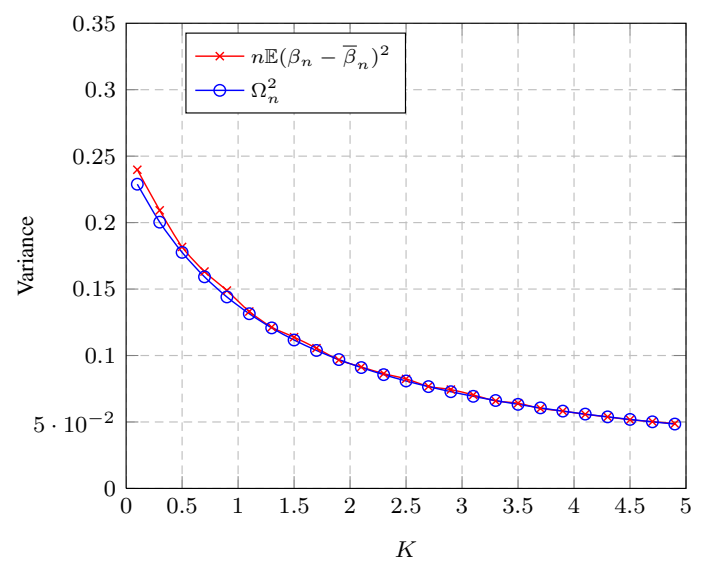

Fig. 2. Empirical and asymptotic variances with respect to the $K$.

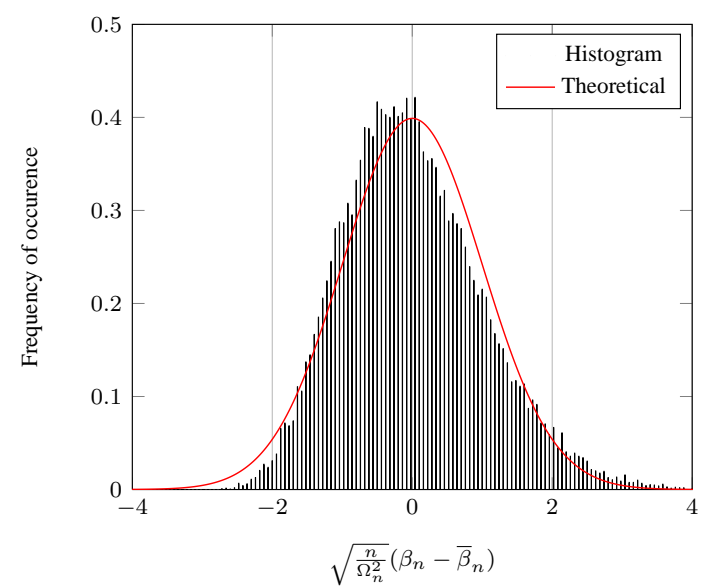

Fig. 3. Histogram of $\sqrt{\frac{n}{\Omega_{n}^{2}}}\left(\beta_{n}-\bar{\beta}_{n}\right)$.
Telecommunications, vol. 10, no. 6, pp. 585-595, Nov/Dec 1999, Technical Memorandum, Bell Laboratories, Lucent Technologies, October 1995.

[2] Foschini G.J., "Layered space-time architecture for wireless communication in a fading environment when using multiple antennas," Bell Labs Technical Journal, vol. 1, no. 2, pp. 41-59, 1996.

[3] D.N.C Tse and S. Hanly, "Linear multi-user receiver: Effective interference, effective bandwidth and user capacity," IEEE Trans. on Information Theory, vol. 45, no. 2, pp. 641-657, Mar. 1999.

[4] D.N.C. Tse and O. Zeitouni, "Linear multiuser receivers in random environments," IEEE Trans. on Information Theory, vol. 46, no. 1, pp. 171-188, Jan. 2000.

[5] A. Kammoun, M. Kharouf, W. Hachem, and J. Najim, "A Central Limit Theorem for the SINR at the LMMSE Estimator Output for Large-Dimensional Signals," IEEE Trans. on Information Theory, vol. 55, no. 11, pp. $5048-5063$, nov. 2009.

[6] J. Dumont, P. Loubaton, S. Lasaulce, and M. Debbah, "On the Asymptotic Performance of MIMO Correlated Rician Channels," in Proceedings of the IEEE International Conference on Acoustics, Speech, and Signal Processing, March 2005.

[7] K. Yip and T. Ng, "A Simulation Model for Nakagamim Fading Channels $m<1$," IEEE Trans. on Communications, vol. 48, no. 2, pp. 214-221, Feb. 2000.

[8] W. Hachem, P. Loubaton, and J. Najim, "Deterministic equivalents for certain functionals of large random matrices," Ann. Appl. Probab., vol. 17, no. 3, pp. 875-930, 2007.

[9] W. Hachem, P. Loubaton, J. Najim, and P. Vallet, "On bilinear Forms Based on the Resolvent of Large Random Matrices," To appear in Annales de l'IHP: Probability and Statistics, 2010.

[10] J. Baxter, R. Jones, M. Lin, and J. Olsen, "SLLN for Weighted Independent Identically Distributed Random Variables," Journal of Theoretical Probability, vol. 17, no. 1, pp. 165-181, 2004.

[11] Z.D. Bai and J.W. Silverstein, "No eigenvalues outside the support of the limiting spectral distribution of large dimensional sample covariance matrices," Annals of Probability, vol. 26, no. 1, pp. 316-345, 1998.

[12] W. Hachem, P. Loubaton, and J. Najim, "A CLT for Information Theoretic Statistics of Gram Random Matrices With a given Variance Profile," Annals of Probability, vol. 18, no. 6, pp. 2071-2130, 2008. 